\title{
Preliminary Method for Surveying the the State of Batteries Ageing in Isolated PV Systems
}

\author{
A. López Agüera ${ }^{1}$, E. Lorenzo $^{2}$, Rute Cortizo ${ }^{1}$ and I. Rodríguez Cabo ${ }^{2}$ \\ ${ }^{1}$ Department of Particle Physics \& Galician Institute of High Energy Physics \\ Astroparticle Group. Santiago de Compostela University \\ South Campus 15702 Santiago de Compostela (Spain) \\ phone:+34 981 563100, fax:+34 981521091, e-mail: lopez@fpaxp1.usc.es, ircabo@fpaxp1.usc.es \\ ${ }^{2}$ Institute of Solar Energy \\ Polytechnic University of Madrid \\ University City 28004 Madrid (Spain) \\ phone: +34 9154410 60, fax:+34 54463 41, e-mail: lorenzo@ies-def.upm.es
}

\begin{abstract}
A battery ageing estimator based on the observed voltage distributions over a one month timescale has been defined and experimentally verified at different set-ups: the 450 PV-stand alone systems operating at the Pierre Auger Observatory and the 40 meteorological stations of Meteo Galicia. The systematic consideration of this indicator has allowed for the detection of prematurely ageing batteries, before they cause a lack of energy supply. This paves the way for a rather simple but still very effective surveillance of the state of health of stand-alone PV systems.
\end{abstract}

Keywords: Energy storage, batteries, ageing, monitoring techniques, online diagnosis

\section{Introduction}

Batteries are costly components of Photovoltaic (PV) systems playing a key role in stand-alone PV systems' performance. Typically, in autonomous PV systems, 30\% or even more of the lifetime costs may be attributed to battery storage [1]. This is mainly because the operating behaviour of lead-acid batteries is affected during their lifetime by different ageing processes: corrosion, sulphation, acid stratification, water loss, loss of active material, etc. Thus, the evaluation of the battery state of health is an important task to preserve the reliability of the energy service provided by a stand-alone PV system. Available literature contains several attempts at directly estimating battery lifetime from previous simulations of operation conditions [2,3,4]. Attemps of simulated cycling test on batteries under conditions resembling the operation of a battery in a PV system have also being made $[5,6]$. However at present, such approaches have not been translated into results of practical application. The intrinsic complexity of the associated models (for example, the dependency of the corrosion rate on the cell voltage), and the extremely long time required for laboratory battery cycling are possible reasons. Whichever the case, information describing experimental battery ageing on PV systems is extremely scarce.
The analysis of the battery voltage distribution, $\mathrm{f}(V)$, has been proposed as a possible tool for surveying the general state of health of PV systems and, in particular, for estimating battery ageing. The key idea is to recognize that battery ageing, whatever ageing mechanism, always means an increase in apparent internal resistance, which in turn means an increase in ohmic voltage drops during charging and discharging. Consequently, assuming constant operation conditions for the PV system (i.e. constancy of solar radiation and energy consumption) the older the battery is, the wider the observed battery voltage distribution must be. That opens the door for estimating the health of a battery by means of analyzing it's voltage time evolution.

Based on this idea, the comparison between expected (normal) and actual battery voltages, in terms of the RMSE (root mean square error), has been proposed as an indicator of battery ageing, offering two attractive features. On the one hand, it is based on only voltage measurements which are particularly easy to obtain. On the other hand, it can be applied in real time, hence allowing for premature detection of anomalous ageing rates. This way effective warning can be established well before lack of energy supply occurs. This proposition, presented in a previous paper [7] was mainly based on theoretical work.

This paper presents how an experimental confirmation of this method can be effectively applied to existing monitored PV installations. The reliability of the method will be estimated using two experimental set-ups: one characterized by constant consumption, some extreme meteorological conditions and clean atmosphere (Pierre Auger Observatory 'PAO', [8],) and another with bumped-like consumption, soft continental or marine weather and an frequently clouded atmosphere with aerosols (Meteo Galicia meteorological stations). 


\section{Experimental set-up}

The first one is the biggest cosmic ray detector array ever deployed. It is an international collaboration between 18 countries and its main objective is the description of the energy flux at the highest energies ever reached.

This observatory is designed as a grid of 1600 isolated detector stations. All these stations are distributed over an area of $60 \times 60 \mathrm{~km}^{2}$ located in the Malargüe high plateau ${ }^{1}$ [8] in Argentina. Both, solar radiation, ambient temperature and humidity conditions are similar on this area. Each station is powered by a $100 \mathrm{Wp}$ stand-alone PV system and is being continuously monitored by means of a data logger and a radio link. Data is recorded and transmitted each 10 minutes to the project headquarter. Not only is cosmic ray data monitored: PV performance parameters are also being monitored. This way, even if the PAO project's scientific goals are not directly related with PV technology, it constitutes a very interesting set-up for PV stand-alone systems research.

Daily energy consumption at each station is $190 \mathrm{Wh}$. Nominal DC voltage is $24 \mathrm{~V}$ and battery capacity is $\mathrm{C}_{20}=$ $105 \mathrm{Ah}$ battery. Batteries are maintenance free (liquid electrolyte) type from Moura (Brazil), specifically devoted to the automobile market (SLI type). Each system comprises two $12 \mathrm{~V}$ batteries, which are being independently monitored. Monthly average daily in plane irradiation during the worst month is $1.6 \mathrm{~kW} / \mathrm{m}^{2}$. Batteries are coupled to a Isofotón PV array of $100 \mathrm{Wp}$, through a SunSaver 10L MorningStart charge regulator. The load is composed by 3 photomultipliers, a low consumption data-logger, which are being continuously powered, and a radio link sending data each 10 seconds to a central receiver at the headquarter of the PAO. All together, these loads are equivalent to a $10 \mathrm{~W}$ constant load.

The second set-up are the PV systems of the 40 Meteo Galicia meteorological stations. They are placed in the region of Galicia (España), and the solar radiation, humidity and temperature is a bit different among some of them. In general, all have a relatively high humidity and a peaked-like current consumption, as well as, less extremely temperatures than in the Pierre Auger Observatory case. The differences enriches the study since the more general the method is the more applicable and reliable it is.

Like in the PAO case, data are recorded every 10 minutes.

\section{Battery charging and discharging. Voltage Distribution Function: the ageing estimator}

In Fig. 1, it is shown the different voltages of a battery during a day and its corresponding statistical distribution. We can distinguish five different regions of interest:

- Deep Discharge region: below $11.8 \mathrm{~V}$

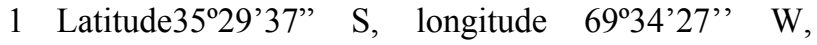
elevation $1430 \mathrm{~m}$. Environmental temperatures ranges between -8 and 30 degrees.
- Discharge region: approximately below $13 \mathrm{~V}$

- Charge-discharge region: over 13V and below 13,5.

- Regulation region: over 13,5V

- Overcharge region: over $15.5 \mathrm{~V}$

The first and the last regions shouldn't be reached in the normal batteries performance.

In this paper, we are only interested on the discharge regime:

When the battery is discharging, its voltages are only fixed by the characteristics of the battery, the consumption and the temperature (not by the regulator, etc.). This led us to take into account only this working region to study the batteries' ageing.

The preliminary method for evaluating a battery's state of ageing [9] is inspired from the original idea presented in [7]. Whatever the type of evolution the battery is having, standard or anomalous, the passage of time creates a gradual loss of capacity or expected lifespan, intrinsically accompanied by an overall increase in internal resistance. This increase in resistance corresponds in principle to a faster discharge of the battery.

The method is based on the fact that the ageing is related with the expansion and slight retraction of the distribution in the discharge region. In other words, the expected variations of the battery voltage distribution's

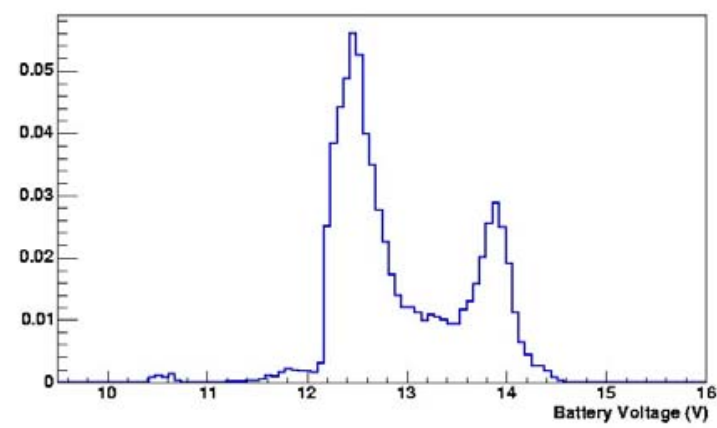

shape.

Fig. 1. Generic Voltage Distribution of a battery

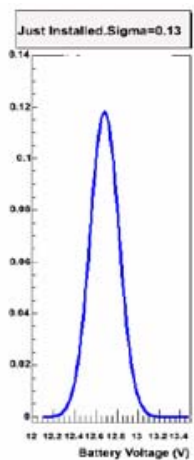

a)

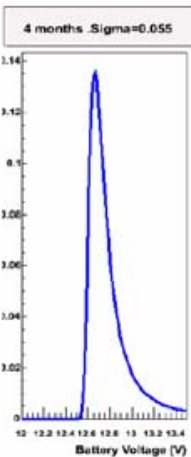

b)

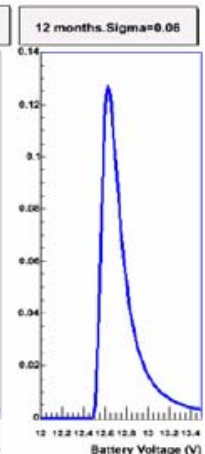

c)

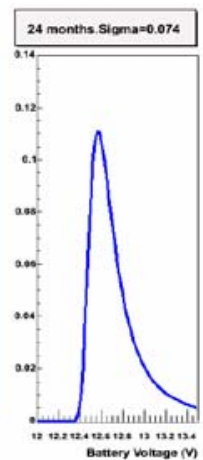

d)
Fig. 2. Experimental voltage distributions of a battery for different months. The horizontal and vertical axis represent, respectively, voltage and density of probability values. Plots $\mathrm{a}, \mathrm{b}, \mathrm{c}$ and $\mathrm{d}$ correspond to months number $1,4,12$ and 24 , after starting of operation. 
As an illustrative example, Fig. 2. shows a observed PAO's battery voltage distributions in discharge regime for a single battery along the timescale. Each plot corresponds to a different month in the battery's life. Two main facts can be observed:

a) During the first months (about 3 months, plot $2 a$ ) the voltage distribution fits a Gaussian shape while in the following it fits a Weibull shape [10]. At present, we do not have a proper physical explanation for this fact. Waiting for further research work, we suspect the initial "Gaussian period" is related to ending formation plate processes, which typically occurs in SLI batteries after having been delivered from factories [11].

b) During the "Weibull period" (plots 2b,c,d) the voltage distribution progressively spreads, which is consistent with the expected battery ageing. Moreover, the maximum occurrence voltage (i.e. the voltage at the peak of distribution) decreases. This is also in coherence with an increase in internal resistance, and therefore with expected battery ageing.

Difference between whichever two distributions can be quantified in terms of the RMSE, defined as:

$$
\mathrm{RMSE}=\sqrt{\sum_{\mathrm{i}=1}^{\mathrm{N}^{\mathrm{o} \text { bins }}} \frac{\left[\mathrm{f}_{\mathrm{A}}\left(\mathrm{V}_{\mathrm{i}}\right)-\mathrm{f}_{\mathrm{R}}\left(\mathrm{V}_{\mathrm{i}}\right)\right]^{2}}{\mathrm{~N}_{\mathrm{V}}}}
$$

where $\mathrm{V}_{\mathrm{i}}$ represents the voltage intervals used to obtain the voltage distribution, $\mathrm{N}_{\mathrm{v}}$ is the total number of intervals, and $f_{R}(V i)$ and $f_{A}\left(V_{i}\right)$ refer to the reference and actual probability densities (or frequencies) of each $\mathrm{V}_{\mathrm{i}}$ interval. In our case, selected values are $\mathrm{N}_{\mathrm{V}}=80$ and $\mathrm{V}_{\mathrm{i}}=20 \mathrm{mV}$.

\section{A) The Pierre Auger Observatory}

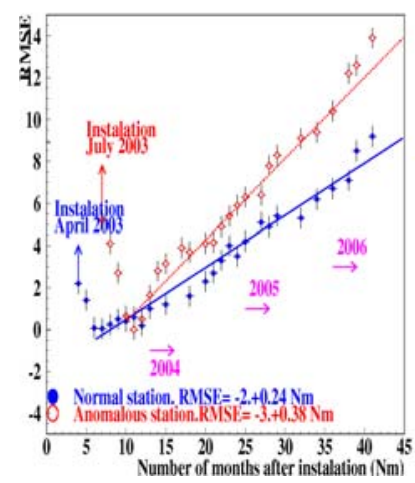

a)

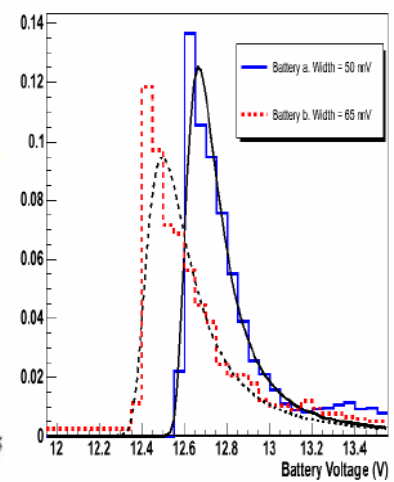

b)
Fig. 3. a) RMSE versus time for two different single PAO's batteries. (b) Voltage distribution of both batteries at the reference month

Fig. 3-a) shows the evolution of the RMSE for 30 months, having roughly corrected the effects of temperature and irradiation, for two single batteries suffering different installation conditions. In both cases, the reference month for RMSE calculations is the one corresponding to the most narrow voltage distribution, which also corresponds to the transition from the Gaussian to the Weibull periods.

Battery "a" was installed just one month after reception at the PAO headquarters, where it was submitted to a previous charging process by means of a standard battery charger. Meanwhile, battery "b" was installed four months after reception and without any pre-charging process. Even though neither of the batteries were initially tested, we suspect that such different conditions should translate into different initial capacities. Because of lower storage time and pre-charging precautions, battery "a" plates most probably reach in-field installation with better general health (more advanced formation $^{2}$, absence of sulphation ...) than the plates of battery "b." This is consistent with the different voltage distribution widths observed at the reference months (see figure 3b); battery "a"'s width is narrower than battery "b." This is also consistent with the different voltage values at the peak of the distributions, battery "a" is higher than battery "b." Moreover, poor initial state of health has been repetitively mentioned as a reason for accelerated battery ageing [12]. This is also consistent with the different tilt of the observed RMSE evolution for both batteries (see figure 2b). We think these reasons suffice to probe how the comparison between reference and actual battery voltages, in terms of the RMSE over a month time scale, can be effectively used as a proper indication of battery ageing.

The reader should note that voltage distributions we have analyzed correspond only to discharge operation $(V<13$ $\mathrm{V})$. This is because voltages during charge operation are significantly affected by other factors besides battery ageing mechanisms. In particular, they are affected by the temperature compensation of the charge regulator set points. It is also worth noting that a quantitative relation between RMSE and battery capacity decrease should exist. In fact, a $10 \%$ increase in RMSE value for each $10 \%$ decrease of capacity has been suggested in the original paper [7]. However, we believe our present experimental baggage does not suffice to asses this

2 "Formation" of the plates' means that the relevant parts of the homogeneous plate materials used in the manufacture of the battery are converted to lead dioxide on the positive electrodes and to sponge lead on the negative electrodes. The plates of new batteries are often incompletely formed and have large remnants of other materials $\left(\mathrm{SO}_{4} \mathrm{~Pb}, \mathrm{PbO}\right)$ which lead to initial capacities that are significantly below the nominal values. This poor formation can normally be compensated with appropriate initial cycles, but in stand-alone PV systems in particular it can not be assumed that this will happen, because of the intrinsically limited availability of charging current. Because of that, it is important to take some provisions to ensure that the capacity of the delivered batteries is close to the nominal value. 
particular point. That remains for future work. Meanwhile, we have applied the RMSE indicator for detection of prematurely aged batteries.

\section{Statistically using of the RMSE}

As mentioned above, batteries at the PAO are type SLI and have sometimes being installed after rather long storage periods. Neither these types of batteries nor this lack of precaution is the best procedure when looking for long battery lifetime. However, it should be understood that very big international projects, as the PAO is, because unavoidable complex associate management sometimes leads to nonoptimal technical decisions. Moreover, battery behavior studies in this project have been undertaken after the installation of the PV systems. That helps to explain why prematurely aged batteries can be found at the framework of one of the most outstanding projects in the rather impressive scene of particle physics research. The good news is that such highly sophisticated experiments pave the way for general technology improvement. For example, associated studies as described here.

Fig. 4. shows the RMSE increase from August 2003 to August 2004 and for 450 batteries. All the batteries were already at the "Weibull period" throughout the year. Because RMSE closely evolves linearly with time at this period, such an increase can be understood as an indicator of the battery ageing rate along this year. Somewhat intuitively, we have established a rude definition of the standard behavior as corresponding to the red lines in the plot.

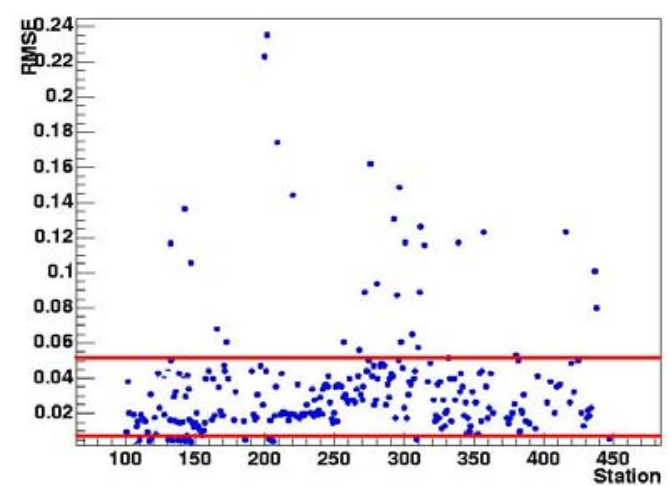

Fig. 4. RSME increase, from August 2003 to August 2004 , for 450 PAO's batteries. Values out of range have been considered as corresponding to anomalous ageing rates.

The 22 batteries falling upon this threshold have been then considered as possible candidates for premature ageing. The interesting point is that when writing this paper (November 2005) 16 of them have been already been changed because they have failed to supply energy to the load. The operational lifetime of most these batteries was lower than two years.
Because of this experience, the use of the RMSE indicator is now being considered for routine maintenance procedure of the 3200 stand-alone PV system that should be installed before June 2007, at the completion of the Pierre Auger Observatory.

\section{Meteo Galicia. First Results}

By the moment, we have only a few results concerning the Meteo Galicia data, but they point in the right direction. It can be observed, as expected and seen in the PAO case, an increase of the discharge distribution function of one battery over three years (see Fig. 5.) as well as a small decrease in its values. We observed this fact in two another recollection of three months, like the Fig. 5. one, as well as a decreasing in its voltage mean values with time.

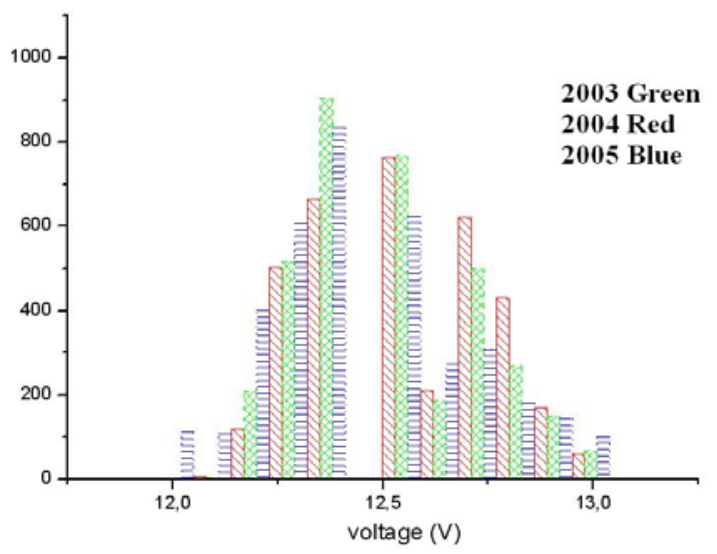

Fig. 5. Voltage discharging distribution function of a

Mete Galicia's battery in three different years (green October 2003, red October 2004, yellow October 2005)

In PAO case, the meteorological conditions of the area allow us to correct simultaneously both the temperature and the irradiation effect, since they behave lineary one each other (see Fig. 6.). In Meteo Galicia case, it should be noticed that the behaviour is different and we do not know yet if such a roughly correction can be done. Further work is needed.
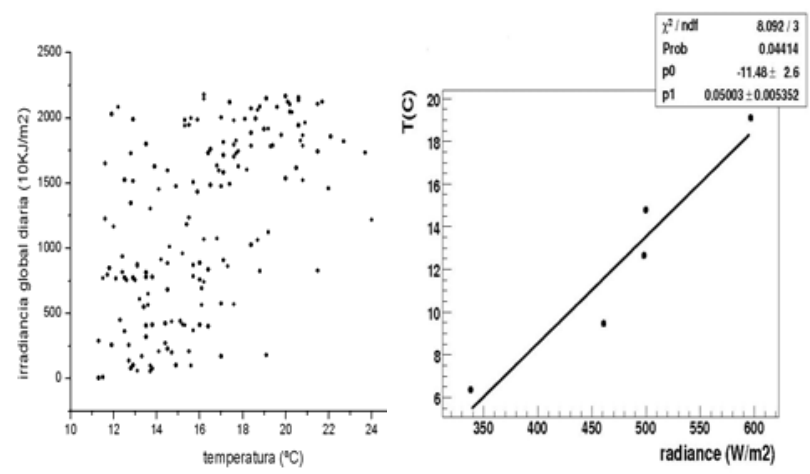

Fig. 6. daily irradiance vs time in a Meteo Galicia station (left) and month mean radiance in Malargüe vs time (right). 


\section{Conclusions}

Premature ageing and PV array degradation are important anomalies that may remain unnoticed for a long time in stand-alone PV systems. This digest has presented a preliminary experimental probe of these anomalies for early detection, before causing a lack of energy supply, by only observing the evolution of the battery's discharging voltages. The key idea is to recognize that battery ageing means an increase in the internal resistance and therefore an increase in voltage drops. Consequently, the older the battery is the wider the observed battery voltage distribution must be. It is quantified by means of the discharge distribution function an in terms of the RMSE.

The Pierre Auger Observatory PV systems are characterized by the constancy of daily energy consumption throughout the year and the Meteo Galicia system is characterized by a peaked consumption and different meteorological conditions. We think that this experimentally procedure could be in brief applied to any stand-alone PV, since that systematic voltage measurements are already being made in many PV systems worldwide.

\section{Acknowledgments}

The authors are grateful to Isofotón for partially support this work and Meteo Galicia (Consellería de Innovación. Xunta de Galicia) for allowing us to explore their experimental data.

\section{References}

[1] Sauer DU. In Electrochemical Storage for Photovoltaics, Handbook of Photovoltaic Science and Engineering, Luque A, Hegedus S (eds). Wiley: Chichester, 2003; pp 799-862
[2] Cherif A, Jraidi M and Dhouib A. A battery ageing model used in stand alone PV systems, Journal of Power Sources 2002; 112: pp 49-53

[3] Chenlo FA. Lifetime and sizing of batteries in stand alone PV plants. Proceedings of the $13^{\text {th }}$ European Photovoltaic Solar Energy Conference, Amsterdam, 1994; pp 408-411

[4] Degner T, Gabler H and Stöcklein A. A model for the ageing of lead-acid batteries in PV systems. Proceedings of the $13^{\text {th }}$ European Photovoltaic Solar Energy Conference, Amsterdam, 1994; pp 422-426

[5] IEEE Guide for Selection, Charging, Test, and Evaluation of Lead-Acid Batteries Used in StandAlone Photovoltaic (PV) Systems. IEEE Std 1361 (2003)

[6] J. Nickoletatos and S. Tselepis. Results of PV and wind simulated tests on batteries. Proceedings of the $20^{\text {th }}$ European Photovoltaic Solar Energy Conference, Barcelona, 2005

[7] E. Lorenzo and S. Labed. Progress in Photovoltaics Vol 13,3, 251-260, (2004.)

[8] A. López Agüera and I. Rodríguez Cabo. "The Pierre Auger Project as a Challenging Tool for Studying PV Systems". Sent to ICREPQ'07

[9] A. López Agüera, E. Lorenzo (IES) and I. Rodríguez Cabo. GAP-2005-108.

[10] A. Papoulis, Probability, Random Variables and Stochastic Processes, McGraw-Hill, New York, (1965)

[11] D. Linden, Handbook of Batteries, McGraw-Hill, New York (1995).A. Jossen, J. Garche and D.U. Sauer, Solar Energy, 76, pp 759-769, (2004)

[12] A. Jossen, J. Garche and D.U. Sauer, Operation conditions of batteries in PV applications, Solar Energy, 76, pp 759-769, (2004) 